\title{
Problemática espacial del sur del departamento del Atlántico y la planeación territorial estratégica*
}

\author{
Efraín Llanos Henríquez. \\ (D) http://orcid.org/0000-0002-9219-2489 \\ Universidad del Atlántico, Colombia
}

DOI: http://dx.doi.org/10.17081/eduhum.19.32.2539

Recibido: 18 de junio de 2016

Aceptado: 23 de septiembre de 2016

\section{Territorial problem in the south of Atlantico area (Colombia), and the territorial strategic planning}

Palabras clave:

Planeación territorial, Problemática espacial, Sur del Atlántico.

Keywords:

Territorial planning,

Spatial problems,

South of the Atlántico.

\begin{abstract}
Resumen
Una parte considerable de los problemas espaciales que afectan a muchos entes territoriales en Colombia se debe en gran medida a la inexistencia de adecuadas políticas de ordenamiento y planeación del territorio. En este trabajo se hace una caracterización de los principales problemas espaciales de los municipios del sur del Atlántico, se analizan los factores que han incidido en su surgimiento y continuidad y se plantean algunas alternativas de solución, desde la Nueva Planeación Territorial Estratégica. El trabajo se sustenta en una amplia utilización de fuentes secundarias (investigación bibliográfica, uso de cartografía - mapas, planos, fotografías e imágenes satelitales- y estadísticas económicas, políticas y sociales, entre otras), así como en la realización de entrevistas y encuestas a habitantes de los municipios del área, en trabajos de campo y en la experiencia personal del autor en la zona estudiada. El trabajo se soporta teóricamente en la concepción del espacio como construcción social, planteada, entre otros, por Santos (2000), en los análisis y métodos de la Geografía Histórica (Tovar, 1986), y en especial en el llamado por Harvey (2003) Materialismo Histórico Geográfico y en los aportes de la Nueva Planeación Territorial Estratégica. Es una investigación de tipo descriptivo y explicativo.
\end{abstract}

\begin{abstract}
The absence of appropriate policies related to territory management and planning become an essential territorial problem, that may affect many of the local authorities in Colombia. This paper focuses on the characterization and factors that influence the emergence and continuity of specific territorial problems and some alternative solutions evidence, based on a New Strategic Territorial Planning in the south of the Atlantico.The research was inspired by extensive secondary resources: (bibliographic research, use of mapping plans, photographs, satellite images and economic, political, and social statistics among others). In addition, interviews and surveys were conducted among inahabitants of the area, and the author's experience in relation to the area studied.

This work focuses also on the concept of territorial area as a social construction, Santos (2000), historical geography analysis and methods (Tovar, 1986), and the Geographical Historical Materialism and Contributions of the New Strategic Territorial Planning by Harvey (2003). This is a descriptive and explanatory research paper.
\end{abstract}

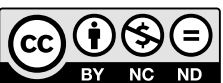

Referencia de este artículo (APA): Llanos, E. (2017). Problemática espacial del sur del departamento del Atlántico y la planeación territorial estratégica. En Revista Educación y Humanismo, 19(32), 159-173. http://dx.doi.org/10.17081/eduhum.19.32.2539

\footnotetext{
* Este artículo de reflexión está asociado al proyecto de investigación: Historia, sociedad, cultura y patrimonio en el Caribe, desarrollado por el Grupo de Estudios Interdisciplinarios sobre el Caribe, de la Universidad Simón Bolívar.

1. Licenciado en Ciencias Sociales y Económicas, Universidad del Atlántico, Colombia. Especialista en Ciencias/Meteorología, Universidad Nacional de Colombia. Magíster y doctor en Geografía, Convenio Universidad Pedagógica y Tecnológica de Colombia (UPTC) e Instituto Geográfico Agustín Codazzi (IGAC). Profesor Titular Universidad del Atlántico y Universidad Simón Bolívar, Colombia efrainllh56@gmail.com
} 


\section{Introducción}

Los municipios que conforman el sur del departamento del Atlántico comprenden una de las zonas de esta unidad político-administrativa que mayor cantidad de problemas socioeconómicos presenta, destacándose entre estos: las Necesidades Básicas Insatisfechas (NBI), que afectan a un considerable porcentaje de su población y hacen que una gran parte viva en condiciones de pobreza y de miseria; además, sus habitantes están expuestos a una serie de riesgos y amenazas naturales y no cuentan con políticas de planeación adecuadas por parte de las administraciones locales y departamentales, que les permitan superar los niveles de pobreza e impedir las consecuencias negativas de los desastres naturales.

Con el objetivo de identificar la problemática espacial que afecta a estos municipios, analizar sus causas y ponderar los aportes que la Planeación Territorial Estratégica puede hacer en la búsqueda de posibles soluciones, el presente artículo pretende responder las siguientes preguntas: ¿Cuáles son los problemas espaciales que afectan a los municipios del sur del departamento del Atlántico?, ¿cuáles son las causas o factores que generan dicha problemática espacial? y ¿qué elementos de la Nueva Planeación Territorial Estratégica (NPTE) pueden contribuir en la búsqueda de soluciones a esta problemática?

El artículo se soporta teóricamente en la concepción del espacio como construcción social, planteada por teóricos de la Geografía
Radical como David Harvey y Milton Santos, y en los fundamentos de la llamada Planeación Territorial Estratégica. El mismo se ha estructurado en cuatro partes: en la primera, se hace una descripción del área de estudio; la segunda aborda los aspectos concernientes a la metodología y los soportes teóricos que la sustentan; la tercera parte, que constituye el eje central del artículo, analiza la problemática espacial de esta zona a partir de la revisión de estadísticas económicas y sociales, de la información primaria obtenida por el investigador, de la interpretación de material cartográfico y de una exhaustiva consulta de materiales bibliográficos; en la cuarta y última parte se identifican los elementos básicos de la NPTE, que pueden contribuir en la búsqueda de soluciones de los problemas que afectan a los municipios del sur del departamento del Atlántico, y como corolario del trabajo se esbozan unas conclusiones.

\section{Área de estudio}

La zona denominada como sur del departamento del Atlántico está constituida por seis municipios: Campo de la Cruz, Candelaria, Manatí, Repelón, Santa Lucía y Suan (ver Figura 1), los cuales cubren un área de $908 \mathrm{~km}^{2}$ (26,8 \% del área departamental). De acuerdo con el DANE, su población a junio 30 de 2010 comprendía 90.276 habitantes $(3,9 \%$ de la población del departamento), para una densidad de 99,42 habitantes $/ \mathrm{km}^{2}$, relativamente baja si se compara con la del total del Atlántico que es de 683,13 habitantes $/ \mathrm{km}^{2}$. El terreno que ocupan es sobre todo plano y forma parte de la llanura de inundación del río Magdalena, por lo tanto está 
conformado por sedimentos de origen fluvial y relativamente recientes. Desde el punto de vista del clima, estos municipios se caracterizan por presentar temperaturas elevadas a lo largo del año $\left(29^{\circ} \mathrm{C}\right.$ en promedio) y precipitaciones de alrededor de $1.200 \mathrm{~mm}$ anuales, repartidas en dos períodos, uno que va de abril a junio y el otro, de agosto a noviembre.

Los municipios del sur del departamento del Atlántico se han constituido en la zona de este ente territorial que mayores problemas espaciales presenta, destacándose lo concerniente a la pobreza en que se encuentra una porción considerable de sus habitantes, los cuales padecen de varias Necesidades Básicas Insatisfechas (NBI), de ahí que sus condiciones de vida no sean las más adecuadas y que su Índice de Calidad de Vida (ICV) este por debajo del promedio nacional, no obstante ser parte del departamento del Caribe colombiano que posee, en este sentido, mejores niveles a escala regional y nacional.

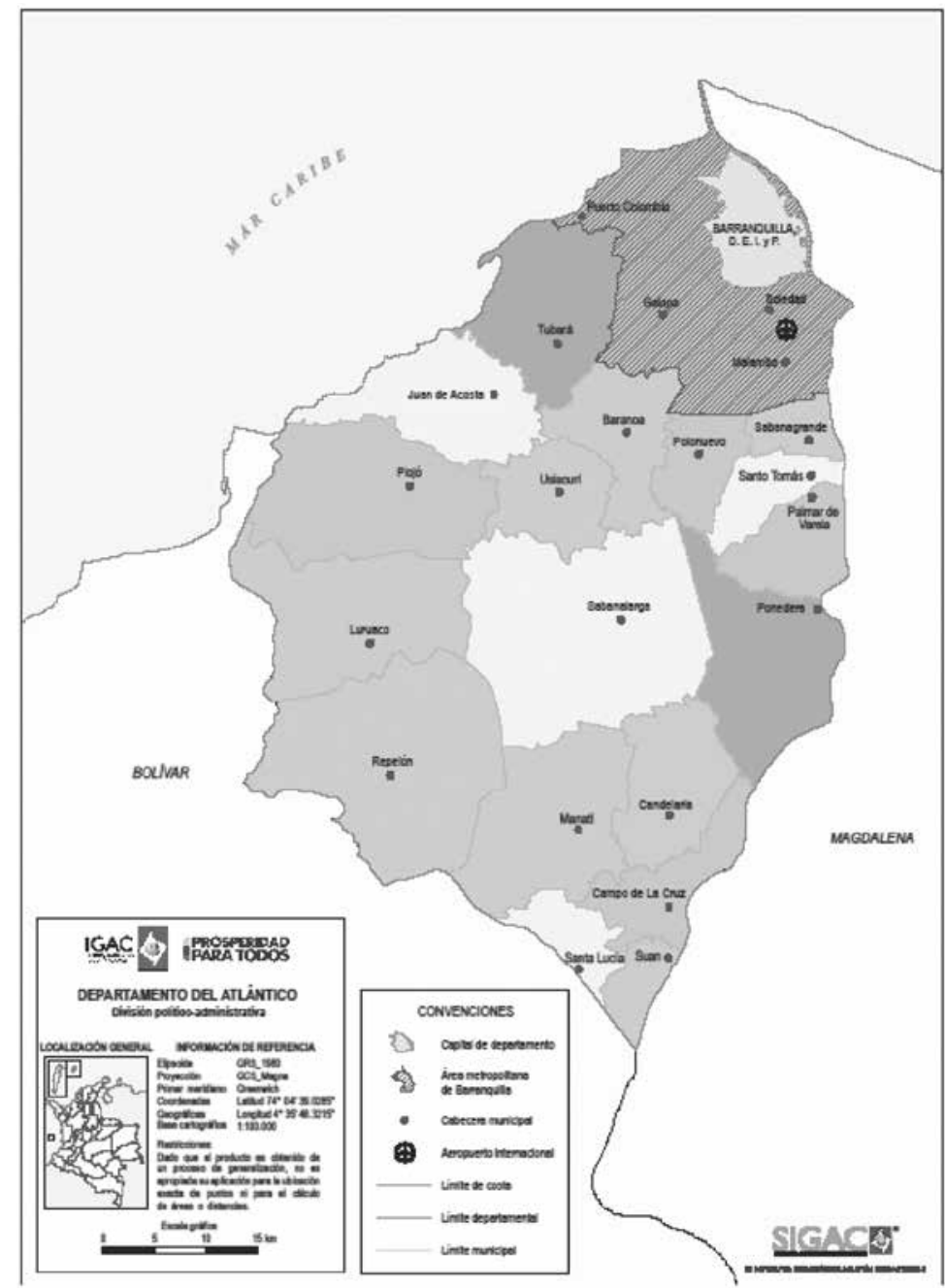

Figura 1. Departamento del Atlántico

Fuente: Instituto Geográfico Agustín Codazzi (2016) 
Entre los factores que más han contribuido a la permanencia e incluso al desmejoramiento de estas condiciones se encuentran la inexistencia de mecanismos de planeación territorial y la persistencia de formas de gestión gubernamental caracterizadas por la ineficiencia y la politiquería. En esta investigación se identifican los principales problemas espaciales que padecen estos municipios, se analizan los factores que inciden en el surgimiento y agravamiento de estas disfuncionalidades y se plantean algunas sugerencias en torno a la Nueva Planeación Territorial Estratégica y su importancia en la solución de los problemas espaciales.

\section{Método}

El trabajo se enmarca en el ámbito de la investigación cualitativa y es de carácter descriptivo y explicativo. Está sustentado en una profunda revisión bibliográfica acerca de la Nueva Planeación Territorial Estratégica (NPTE), con autores como: Farinós (2007, 2009), Iracheta (1997), Fernández (2006) y Gómez Orea (2007); asimismo se sustenta en la concepción del espacio como construcción social, planteada entre otros investigadores, por Harvey (2003) y Santos (2000). Asimismo, las estadísticas económicas y sociales referidas a los municipios de esta zona se constituyen en elementos indispensables para el desarrollo de la investigación, así como los planos, mapas e imágenes de esta región del departamento del Atlántico. Por último, las observaciones de campo, las entrevistas y encuestas aplicadas a habitantes de estos municipios y las experiencias personales del autor también se erigen en sustentos básicos de esta investigación.

\section{La problemática espacial del sur del depar-} tamento del Atlántico

De acuerdo con Llanos (2007), "los problemas espaciales se conciben como un conjunto de condiciones anormales, tanto de orden físico como social, que se generan en un lugar o territorio, como consecuencia de factores de diversa índole, ya sean económicos, políticos, sociales, etc." (p.12). En tal sentido, los municipios que conforman el sur del Atlántico padecen una serie de condiciones adversas que hacen que su problemática espacial sea una de las más agudas, especialmente desde el punto de vista social, y que los han constituido en una de las zonas que presenta unos indicadores de Necesidades Básicas Insatisfechas (NBI) más elevados. Por lógica, los Índices de Desarrollo Humano (IDH) no son los más alentadores; y en concreto, aunque de este indicador no existen cifras municipales, se puede deducir que su nivel no es el más favorable, si se tiene en cuenta que los tres elementos que se utilizan para hacer el cálculo, es decir, una vida larga y saludable, el acceso al conocimiento y un nivel de vida digno, no son muy positivos en estos municipios que conforman esta zona del departamento. Las condiciones de pobreza de estas poblaciones se pueden observar en las Tablas 1 y 2.

Como se evidencia en los cuadros siguientes, uno de los problemas espaciales más notorios que tienen los municipios que conforman el sur del Atlántico es la pobreza y la miseria, que afecta a la mayor parte de su población $(60,32$ $\%$ de sus habitantes son pobres y el 38,78\% vive en condición de miseria) y que se refleja 
Tabla 1. Necesidades básicas insatisfechas y sus componentes en los municipios del sur del Atlántico a 31 de diciembre de 2011

\begin{tabular}{|l|c|c|c|c|c|c|c|}
\hline Municipio & $\begin{array}{c}\text { Población } \\
\text { con NBI }\end{array}$ & $\begin{array}{c}\text { Población en } \\
\text { miseria }\end{array}$ & $\begin{array}{c}\text { Componente } \\
\text { vivienda }\end{array}$ & $\begin{array}{c}\text { Componente } \\
\text { servicio }\end{array}$ & Hacinamiento & $\begin{array}{c}\text { Inasistencia } \\
\text { escolar }\end{array}$ & $\begin{array}{c}\text { Dependencia } \\
\text { económica }\end{array}$ \\
\hline Campo de la Cruz & 62,86 & 35,79 & 14,30 & 43,13 & 15,50 & 10,78 & 30,17 \\
\hline Candelaria & 74,22 & 35,85 & 4,52 & 57,19 & 23,55 & 3,10 & 36,39 \\
\hline Manatí & 57,14 & 22,33 & 4,65 & 36,15 & 9,81 & 2,41 & 31,73 \\
\hline Repelón & 52,33 & 24,47 & 15,79 & 32,88 & 8,96 & 2,36 & 25,94 \\
\hline Santa Lucía & 60,0 & 30,93 & 15,63 & 35,55 & 20,13 & 6,37 & 33,21 \\
\hline Suan & 55,4 & 23,31 & 12,56 & 5,52 & 24,01 & 2,33 & 38,01 \\
\hline
\end{tabular}

Fuente: http://www.dane.gov.co/index.php/estadisticas-por-tema/pobreza-y-condiciones-de-vida/necesidades-basicas-insatisfechas-nbi. Elaboración del autor

Tabla 2. Otros indicadores sociales de los municipios del sur del departamento del Atlántico

\begin{tabular}{|l|c|c|c|c|c|c|}
\hline \multicolumn{1}{|c|}{ Municipio } & $\begin{array}{c}\text { Cobertura } \\
\text { Acueducto } \\
\mathbf{( \% )}\end{array}$ & $\begin{array}{c}\text { Continuidad en el } \\
\text { servicio en horas }\end{array}$ & $\begin{array}{c}\text { Cobertura } \\
\text { Alcantarillado } \\
\mathbf{( \% )}\end{array}$ & $\begin{array}{c}\text { Cobertura } \\
\text { Energía (\%) }\end{array}$ & $\begin{array}{c}\text { Analfabetismo } \\
\text { en población } \\
\text { mayor de 15 años }\end{array}$ & $\begin{array}{c}\text { Analfabetismo } \\
\text { en población } \\
\text { mayor de 5 años }\end{array}$ \\
\hline Campo de la Cruz & 82,8 & 12 & 1,5 & 90,1 & 20,9 & 17,2 \\
\hline Candelaria & 61,3 & 6 & 9,1 & 96,2 & 26,5 & 22,5 \\
\hline Manatí & 68,3 & 6 & 0,5 & 89,8 & 17,8 & 14,4 \\
\hline Repelón & 82,0 & 24 & 0,4 & 96,4 & 17,3 & 15,4 \\
\hline Santa Lucía & 85,7 & 12 & 27,1 & 93,8 & 18,3 & 16,6 \\
\hline Suan & 90,8 & 12 & 0,5 & 97,9 & 17,3 & 14,6 \\
\hline
\end{tabular}

Fuente: http://www.camarabaq.org.co/images/pdf/atlantico\%20en\%20cifras\%202009.pdf

http://www.dane.gov.co/daneweb_V09.Gobernación del Atlántico (2012). Análisis de la situación de la salud. Elaboración del autor.

en la alta dependencia económica, en la inasistencia escolar, en el hacinamiento crítico, en las condiciones no aptas de las viviendas y en los deficientes servicios públicos que reciben, en especial los relacionados directamente con la salud de sus habitantes, es decir, los servicios de acueducto y alcantarillado. De idéntica manera, el nuevo Índice de Pobreza Multidimensional (basado en tres aspectos: educación, salud y calidad de vida) muestra que los municipios del sur del Atlántico presentan el mayor porcentaje de población pobre, con un promedio para los seis municipios de $84,7 \%$ de acuerdo con cifras del Departamento Nacional de Planeación, citadas por la Gobernación del departamento del Atlántico.
Aunque las cifras referidas al servicio de acueducto muestran una cobertura promedio de $78,48 \%$, en las entrevistas realizadas, los habitantes afirman que su calidad y frecuencia dejan mucho que desear, ya que el agua no es potable y en muchas ocasiones presenta sedimento y partículas muy visibles, que los obliga a hervirla para poder consumirla o a usarla para lavar. Además, manifiestan que la frecuencia en la prestación del servicio no es la más adecuada, en algunos lugares, por ejemplo, se presta el servicio pocas horas al día y en determinados casos, algunos días a la semana. Las cifras del alcantarillado reflejan claramente la casi inexistencia de este servicio, incluso en 
el caso de Santa Lucía que, según las cifras del DANE, cuenta con una cobertura de $27,1 \%$. Sin embargo, nuestra visita a este municipio mostró cómo en gran parte de sus calles corren las aguas negras. Aunque las obras de recuperación adelantadas con motivo de la inundación del año 2010 han implicado una mejoría en algunos de estos servicios, la situación sigue siendo deficitaria.

La incidencia de la mala calidad de los servicios de agua y alcantarillado en la salud de los habitantes del sur del Atlántico se refleja en la presencia de enfermedades relacionadas con la deficiencia de estos servicios, tales como Enfermedad Diarreica Aguda (EDA), parasitosis intestinal y dengue, tanto clásico como hemorrágico, lo cual conduce a que en estos municipios, "Las tasas de mortalidad infantil, materna y neonatal se encontraron por debajo del indicador nacional con los siguientes valores: 15,3 por 1.000 nacidos, 60,4 por 100.000 nacidos vivos y 2,5 por 1.000 nacidos vivos, respectivamente" (Navarro, 2011, p.3) y afectan más que todo a la población de menores recursos de estos municipios. Incluso, para el año 2016, estas tasas siguen mostrando un comportamiento desfavorable en comparación con los promedios departamental y nacional.

Todo este problema de la salud se ve más agravado aún por la carencia de una infraestructura de servicios de salud adecuada. Según cifras de la Secretaría de Salud del departamento, la zona cuenta con 4 hospitales, 4 centros de salud y 8 puestos de salud; sin embargo, estos se caracterizan por mostrar deficiencias en cuanto a la infraestructura física, la dotación hospitalaria y la atención de los pacientes. Este último problema se encuentra más que todo en las áreas rurales, donde los médicos solo atienden pocas horas en el día. Igualmente, la salud de los habitantes del sur del departamento, ya de por sí deficiente, se agravó aún más con las inundaciones que se presentaron a finales del año 2010 y cuyas secuelas se siguen manifestando incluso hoy.

Como se sabe, la educación es uno de los elementos fundamentales en la lucha contra la pobreza y la miseria, ya que al alcanzar mejores niveles educativos las posibilidades de acceder a un mejor empleo son más reales y, por ende, a un mejor nivel de vida; sin embargo, en la zona sur del departamento del Atlántico, las tasas de analfabetismo están por encima del promedio nacional y departamental $(8,9 \%$ y $7,1 \%$ para mayores de 5 años, y 8,4 \% y 6,6\% para mayores de 15 años, respectivamente), tal como se puede notar en la Tabla 2, lo cual refleja que el acceso a la educación es vedado a un sector notable de la población, y por lo tanto a la posibilidad de que este alcance mejores niveles de ingreso y de vida.

El problema de la educación se agrava aún más, al constatar que ni siquiera quienes tienen acceso al sistema educativo podrán conseguir empleos de buena calidad y bien remunerados, debido al bajo nivel que presentan las instituciones educativas de los municipios de esta zona 
del departamento y que se manifiesta nítidamente en los pobres resultados alcanzados en las pruebas ICFES. Con ello se demuestra que dadas las precarias condiciones de estas instituciones, en ellas no se logra la calidad de la educación, "un compromiso de carácter ético con la sociedad" (Peñaloza, 2008, p.189). Los resultados de los colegios de estos municipios en lo correspondiente al Núcleo Común para los años 2006, 2010 y 2012 se muestran en las Tablas 3 y 4 .

El desempeño mediocre de las instituciones educativas en las Pruebas Saber 11 durante los años señalados, se acentuó aún más después de la inundación producida por el desbordamiento del Canal del Dique, a finales del año 2010, ya que en este año lectivo se mostró un mejor desempeño ( $\sin$ superar la mediocridad) en comparación al año 2006, pero en el año 2013 retrocedió a niveles incluso inferiores a los del penúltimo de los años mencionados. Este desempeño conduce a que las categorías alcanzadas por los colegios de estos municipios se encuentren en su mayor parte en los niveles inferior y bajo, con la excepción de una institución educativa de Repelón, que esta en el nivel medio, de acuerdo con la información más reciente entregada por el ICFES (Tabla 5).

Tabla 3. Resultados Pruebas Saber 11, Núcleo Común, para Campo de la Cruz, Candelaria y Manatí años 2006, 2010, 2012

\begin{tabular}{|l|c|c|c|c|c|c|c|c|c|}
\hline & \multicolumn{3}{|c|}{ Campo de la Cruz } & \multicolumn{3}{c|}{ Candelaria } & \multicolumn{3}{c|}{ Manatí } \\
\hline & $\mathbf{2 0 0 6}$ & $\mathbf{2 0 1 0}$ & $\mathbf{2 0 1 2}$ & $\mathbf{2 0 0 6}$ & $\mathbf{2 0 1 0}$ & $\mathbf{2 0 1 2}$ & $\mathbf{2 0 0 6}$ & $\mathbf{2 0 1 0}$ & $\mathbf{2 0 1 2}$ \\
\hline Biología & 42,66 & 45,82 & 38,96 & 43,15 & 45,24 & 39,23 & 43,01 & 45,26 & 38,18 \\
\hline Ciencias Sociales & 37,53 & 43,82 & 37,31 & 38,77 & 45,28 & 38,24 & 37,96 & 44,70 & 35,20 \\
\hline Filosofía & 40,74 & 46,60 & 35,38 & 44,72 & 46,19 & 35,10 & 43,54 & 46,98 & 33,93 \\
\hline Física & 44,58 & 46,60 & 40,81 & 45,18 & 45,50 & 41,33 & 37,80 & 46,65 & 40,09 \\
\hline Lenguaje & 43,04 & 44,34 & 40,68 & 43,77 & 44,65 & 41,86 & 39,66 & 46,85 & 39,44 \\
\hline Matemática & 41,35 & 45,28 & 39,76 & 41,20 & 43,78 & 39,19 & 42,44 & 45,26 & 37,88 \\
\hline Química & 42,55 & 46,90 & 41,07 & 42,84 & 45,38 & 41,29 & 39,51 & 47,50 & 40,70 \\
\hline
\end{tabular}

Fuente: www.icfesinteractivo.gov.co. Elaboración del autor

Tabla 4. Resultados Pruebas Saber 11, Núcleo Común, para Repelón, Santa Lucía y Suan, años 2006, 2010, 2012

\begin{tabular}{|l|c|c|c|c|c|c|c|c|c|}
\hline & \multicolumn{3}{|c|}{ Repelón } & \multicolumn{3}{c|}{ Santa Lucía } & \multicolumn{3}{c|}{ Suan } \\
\hline & $\mathbf{2 0 0 6}$ & $\mathbf{2 0 1 0}$ & $\mathbf{2 0 1 2}$ & $\mathbf{2 0 0 6}$ & $\mathbf{2 0 1 0}$ & $\mathbf{2 0 1 2}$ & $\mathbf{2 0 0 6}$ & $\mathbf{2 0 1 0}$ & $\mathbf{2 0 1 2}$ \\
\hline Biología & 44,20 & 45,63 & 40,31 & 43,45 & 44,81 & 37,65 & 42,66 & 46,19 & 41,36 \\
\hline Ciencias Sociales & 39,59 & 45,94 & 39,14 & 37,85 & 48,93 & 37,42 & 38,78 & 44,92 & 39,91 \\
\hline Filosofía & 43,33 & 46,34 & 35,36 & 40,84 & 43,59 & 35,17 & 43,07 & 47,95 & 37,65 \\
\hline Física & 42,32 & 45,77 & 41,49 & 43,47 & 45,57 & 40,18 & 42,07 & 46,82 & 42,09 \\
\hline Lenguaje & 44,35 & 43,26 & 41,12 & 44,60 & 44,23 & 41,09 & 44,43 & 46,40 & 43,34 \\
\hline Matemática & 42,36 & 43,97 & 39,53 & 41,75 & 45,55 & 38,43 & 41,60 & 45,54 & 41,29 \\
\hline Química & 43,28 & 45,67 & 42,11 & 43,01 & 48,04 & 40,39 & 43,16 & 47,27 & 43,17 \\
\hline
\end{tabular}

Fuente: www.icfesinteractivo.gov.co. Elaboración del autor 
Tabla 5. Categorías del ICFES en colegios sur del Atlántico años 2006, 2010 y 2013

\begin{tabular}{|c|c|c|c|}
\hline Municipio & $\begin{array}{c}\text { Categoría ICFES } \\
2006\end{array}$ & $\begin{array}{c}\text { Categoría ICFES } \\
2010\end{array}$ & $\begin{array}{c}\text { Categoría ICFES } \\
2013\end{array}$ \\
\hline Campo de la Cruz & 2 inferiores, 3 bajos & 3 inferiores, 2 bajos & 4 inferiores, 1 bajo \\
\hline Candelaria & 1 bajo, 1 medio & 1 inferior, 1 bajo & 1 inferior \\
\hline Manatí & 2 inferiores, 2 bajos & 1 inferior, 2 bajos & 2 inferiores \\
\hline Repelón & 1 inferior, 1 bajo, 1 medio & 2 inferiores, 1 bajo & 3 inferiores, 1 medio \\
\hline Santa Lucía & 1 inferior, 2 bajos & 2 inferiores, 1 bajo & 1 bajo \\
\hline Suan & 1 bajo & 1 inferior,1 bajo & 1 bajo \\
\hline
\end{tabular}

Fuente: www.icfesinteractivo.gov.co. Elaboración del autor

Los anteriores resultados conducen a que, una vez terminada su formación media y vocacional, los estudiantes del sur del Atlántico vean restringido el acceso a la educación superior y, por lo tanto, la posibilidad de que obtengan un título profesional sea remota. De modo que solo les queda la alternativa de vincularse al proceso productivo como empleados y obreros con poca remuneración o, peor aún, convertirse en parte de los desempleados y subempleados que abundan en estos municipios o migren para buscar mejor fortuna en otros lugares del país o del extranjero, en especial, en Venezuela; aunque en la actualidad la situación de esta última opción es diferente, como se analizará en párrafos posteriores.

Otro elemento que agrava la situación social de los habitantes del sur del Atlántico es la existencia notable del desempleo y del subempleo. De esta manera, aunque no existen estadísticas acerca de estos fenómenos, en las entrevistas realizadas, se muestra que uno de los problemas más destacados, aparte del desempleo y el subempleo, es la precariedad del empleo existente y sus condiciones -es temporal, mal remunerado, sin seguridad social, entre otras características. El problema del desempleo es ostensible en la presencia de personas en edad productiva en las esquinas de los municipios en horario laboral; asimismo, el subempleo se observa fácilmente en el tipo de actividades informales que se realizan, dentro de las cuales el mototaxismo y la venta de minutos de celular son las más notorias.

Aunque el problema de la pobreza y de la miseria es multicausal y por ende, hay muchos factores que lo generan, la inexistencia de adecuadas políticas de planeación del territorio, se convierte para el caso de estos municipios atlanticenses en una de las más influyentes, pues, el espacio es una construcción social que responde muchas veces a los intereses y orientaciones de quienes detentan el poder y, en el caso concreto que nos ocupa, las administraciones municipales han respondido más a los intereses de los grupos políticos que representan que a los intereses generales de las comunidades.

La ineficiencia de las administraciones municipales que comprenden esta zona se pone 
de presente al analizar el Indicador de Desempeño Fiscal -que mide la eficiencia en la administración de los recursos de las alcaldías municipales-, notándose que en términos generales dichas administraciones son ineficientes en este aspecto; para el año 2012, en efecto, cuatro de los seis municipios analizados se ubicaban en los últimos lugares a nivel departamental y a nivel nacional; otro se halla en un lugar posterior pero no tan relegado y solo uno se localiza entre los primeros 400 municipios del país; esta situación general no cambia mucho para el año 2013, destacándose el avance de Repelón y el retroceso notorio de Suan (Tabla 6). Lógicamente, esta ineficiencia se traduce en malversación de los recursos, en pocas inversiones y soluciones a los múltiples problemas sociales que inciden sobre estos municipios.
Desempeño Fiscal menor de 60 y mayor de 40. Estas cifras nos muestran claramente que, desde el punto de vista administrativo, las autoridades de estos entes territoriales no cumplen con los requisitos básicos de una buena gestión.

Toda esta situación negativa, tanto desde el punto de vista económico como social, que afecta a los municipios del sur del Atlántico, se ha visto agravada en los últimos tiempos debido especialmente a dos causas: el desastre "natural" (producto de la ineficiente previsión y planeación) que produjo el rompimiento de la carretera y la posterior inundación de las aguas del Canal del Dique en noviembre del año 2010 y el regreso masivo de población de estos municipios que, debido a la negativa situación

Tabla 6. Índice de Desempeño Fiscal municipios del sur del departamento del Atlántico, años 2012 y 2013

\begin{tabular}{|l|c|c|c|c|c|c|}
\hline \multirow{3}{*}{ Municipios } & \multicolumn{3}{|c|}{$\mathbf{2 0 1 2}$} & \multicolumn{3}{c|}{$\mathbf{2 0 1 3}$} \\
\cline { 2 - 7 } & $\begin{array}{c}\text { Indicador de } \\
\text { Desempeño Fiscal }\end{array}$ & $\begin{array}{c}\text { Posición } \\
\text { nacional }\end{array}$ & $\begin{array}{c}\text { Posición } \\
\text { departamental }\end{array}$ & $\begin{array}{c}\text { Indicador de } \\
\text { Desempeño Fiscal }\end{array}$ & $\begin{array}{c}\text { Posición } \\
\text { nacional }\end{array}$ & $\begin{array}{c}\text { Posición } \\
\text { departamental }\end{array}$ \\
\hline Campo de la Cruz & 56,90 & 1.016 & 19 & 60,04 & 951 & 20 \\
\hline Candelaria & 55,42 & 1.044 & 20 & 59,69 & 972 & 21 \\
\hline Manatí & 60,86 & 852 & 16 & 63,07 & 769 & 17 \\
\hline Repelón & 50,19 & 1.088 & 23 & 69,34 & 395 & 10 \\
\hline Santa Lucía & 51,71 & 1.085 & 22 & 55,76 & 1.052 & 23 \\
\hline Suan & 69,70 & 331 & 6 & 62,78 & 790 & 18 \\
\hline
\end{tabular}

Fuente: Departamento Nacional de Planeación

Los Indicadores de Desempeño Fiscal alcanzados por estos municipios en el año 2013 evidencian que cuatro de los seis municipios analizados corresponden a la categoría de Vulnerables, es decir, que presentan un indicador inferior a 70 ; mientras que los dos restantes corresponden a la categoría de En Riesgo, con social había emigrado hacia Venezuela y que hoy, dadas las dificultades por las que pasa este país, han retornado a sus lugares de origen.

La inundación producida por el rompimiento del muro de contención del Canal del Dique significó el ingreso de 2.200 millones de metros 
cúbicos de agua (lo que equivale a un poco más del $50 \%$ del total de las aguas correspondientes a los 20 lagos y lagunas más grandes de Colombia), amén de la destrucción de aproximadamente 35.000 hectáreas de tierras productivas, la afectación a más de 100.000 habitantes del sur del departamento, la destrucción de hospitales, colegios y carreteras, la destrucción o afectación de 10.768 viviendas y la muerte de más 7.000 cabezas de ganado, de acuerdo con las cifras de fuentes gubernamentales y privadas citadas por Sánchez (2011). De los seis municipios del sur del departamento, dos (Santa Lucía y Campo de la Cruz) quedaron totalmente inundados y el resto lo estuvieron parcialmente. Toda esta situación significó un agravamiento de las condiciones ya de por sí precarias de los habitantes de estos municipios.

Por otra parte y como ya se mencionó, el regreso masivo de población de estos municipios procedente de Venezuela ha desmejorado aún más las condiciones sociales de los habitantes del sur del departamento del Atlántico. Aunque hasta el momento (agosto de 2016) no existen cifras oficiales al respecto, el alcalde de Campo de la Cruz, José de León Marenco, manifestó que a su municipio habían ingresado unas 2.500 personas procedentes del país vecino, y en Candelaria se informa de manera extraoficial que han regresado aproximadamente 1.500 personas de Venezuela. Estas dos cifras representan el $17,77 \%$ de la población del primero de los municipios y el 15,67\% del segundo (calculado con base en la proyección de población para el año 2015 efectuada por el DANE).
Este regreso masivo a unos entes territoriales caracterizados por una estructura productiva que no da cabida a la Población Económicamente Activa (PEA), la cual se dedica en un alto porcentaje a actividades propias de la economía informal y cuyo nivel de vida se caracteriza por un alto número de NBI, ha ocasionado el empeoramiento de estas condiciones sin que hasta el año 2016 se hayan diseñado políticas o programas para solucionarlas.

La ineficiente planeación territorial es una de las razones que explica la situación desfavorable en la cual vive la mayor parte de los habitantes de esta zona; aunque la Ley 388 de 1997 obliga a estos entes territoriales a la presentación de Esquemas de Ordenamiento Territorial (EOT), su lectura nos muestra una cantidad de buenas intenciones, propuestas hace mucho tiempo, que no se han llevado a la realidad, verbigracia: la creación de un núcleo territorial integrado por los municipios de Campo de la Cruz, Manatí, Santa Lucía y Suan, que propendiera por la especialización de cada uno de estos municipios. Además, la existencia de los EOT no ha significado un cambio radical en la forma de administrar los municipios, que siguen mostrando las mismas falencias en estos aspectos, con muy pocas excepciones. De igual manera, si bien es cierto que los planes de desarrollo implementados con el apoyo de los gobiernos departamental y nacional constituyen un avance, siguen siendo en general un catálogo de buenas intenciones, cuyo cumplimiento está lejos de realizarse. De esta forma, se puede concluir que la adecuada planeación terri- 
torial sigue siendo la gran ausente, no obstante la existencia de los EOT y los planes de desarrollo.

\section{La planeación territorial estratégica} como aporte a la solución de la problemática espacial del sur del Atlántico

Aunque, como ya se ha anotado anteriormente, los municipios del sur del Atlántico están obligados por la Ley 388 de 1997 a presentar EOT, estos intentos de planeación no han sido hasta ahora los más afortunados, y las condiciones de la mayoría de los habitantes de estos entes territoriales siguen siendo desfavorables. Ante esta situación la Nueva Planeación Territorial Estratégica (NPTE) se erige en una alternativa de planeación y de solución a los múltiples problemas que agobian a esta zona, debido a que, de acuerdo con lo planteado por Fernández (2006) y Farinós (2007, 2010), la NPTE busca la equidad, la competitividad, la sostenibilidad y la gobernanza, entre otras metas, que aplicadas al sur del Atlántico ayudarían a construir un espacio mucho más justo y, por ende, con menos problemas sociales.

La NPTE presenta, en efecto, una serie de características que podrían contribuir en la búsqueda de soluciones de la grave problemática espacial que incide en la deficiente calidad de vida de los habitantes del sur del departamento del Atlántico. Dentro de estas características, y de acuerdo con Llanos (2010), se puede considerar que las siguientes son fundamentales para este propósito:
- Implica una nueva forma de gestión gubernamental, la gobernanza, centrada en la acción colectiva a través de la participación y cooperación de todos los actores y fundamentada en los principios de: participación, apertura, transparencia, responsabilidad, efectividad y coherencia, es decir, todo lo contrario a la gestión municipal actual caracterizada por su ineficacia y corrupción. La aplicación de la gobernanza traería la construcción de un modelo de sociedad mucho más justo y sostenible.

- Es participativa, lo cual significa que todos los grupos de la población tienen la posibilidad de intervenir en el proceso de planeación, desde los dirigentes políticos, los académicos y los técnicos hasta las comunidades; con esto se podría superar uno de los más notorios inconvenientes de la planeación en el sur del Atlántico, la escasa participación de la comunidad en la misma.

- Es de largo plazo, por lo cual se puede evaluar de manera constante e ir corrigiendo los errores que se presentan. Además, se puede adaptar a los ciclos más cortos, correspondientes a los períodos de las autoridades administrativas y gubernamentales de estos entes territoriales.

- Es holística e integral y, por tanto, tiene en cuenta todos los aspectos de cada uno de los territorios, desde el aspecto físico, pasando por los aspectos económicos, políticos, sociales y culturales, todo lo cual conduce a una caracterización, análisis y ponderación de las potencialidades, limitaciones y alterna- 
tivas con las que cuenta esta parte del departamento.

- Es multiescalar, es decir, abarca desde los espacios locales hasta los regionales, nacionales e incluso supranacionales; en este sentido, para el sur del departamento del Atlántico es de vital importancia, ya que permite la integración de los territorios y la búsqueda de soluciones a problemas comunes a los diferentes municipios en consecuencia, teniendo en cuenta las características similares que presentan estos municipios en los aspectos físico, económico, social y cultural y los parecidos problemas espaciales que padecen; a partir de la NPTE se puede proponer la integración de estos municipios en una subregión que facilite la solución de los problemas y procure unas formas de gestión político-administrativas más eficaces.

Por todo lo planteado, las anteriores características de la NPTE se pueden constituir en elementos fundamentales para la búsqueda de la construcción de espacios más equitativos y sostenibles en el sur del departamento del Atlántico.

\section{Conclusiones}

La construcción social de los espacios geográficos responde especialmente a los intereses, necesidades y propósitos de los grupos que detentan el poder; en tal sentido, la construcción del espacio geográfico de la zona correspondiente al sur del departamento del Atlántico ha sido realizada en función de los intereses de unas élites económicas, políticas y sociales, las cuales asociadas, a su vez, a élites departamentales y nacionales, han sacado provecho de esta construcción en desmedro de la mayor parte de la población, y beneficiando solo a los grupos privilegiados. De allí que los municipios del sur del Atlántico pertenezcan a una de las zonas de este ente territorial que mayor cantidad de problemas espaciales presenta, destacándose en especial, la pobreza y la miseria que afectan a la mayor parte de sus habitantes y se reflejan en la presencia de múltiples NBI, en los bajos niveles de salud $\mathrm{y}$ escolaridad, en las precarias condiciones del empleo formal y en el creciente desempleo y subempleo que los agobia. Esta situación se ha agudizado a causa de la inundación que afectó a estos municipios a finales del año 2010 y a la llegada masiva de población que había migrado anteriormente a territorio venezolano.

Aunque las causas de la pobreza y la miseria son muchas, en particular se mencionan las relacionadas con la inequitativa distribución de la tierra, la inexistencia de una estructura económica que garantice un empleo digno y formal a la población trabajadora y la falta de una educación de calidad que posibilite la movilidad social; la inexistencia de adecuadas formas de planeación territorial se considera uno de los factores que más incide en su surgimiento y permanencia, ya que una gestión y ordenamiento del territorio inadecuados afectan negativamente las condiciones de vida de la población. La poca planeación territorial que se hace no responde a los intereses, problemas y necesidades de la mayor parte de la población, sino que, tal como 
lo plantea Harvey (2003), esta planeación lo que busca es garantizar la acumulación y la reproducción del capital.

No obstante esta realidad, existen mecanismos de planeación para solucionar muchos de los problemas espaciales, aun preservando los intereses de la élite. En tal sentido, la NPTE se constituye en una importante alternativa, dadas sus características principales, como son: la gobernanza, que implica una mayor participación de los diferentes grupos sociales en los asuntos de la administración; la participación de las comunidades en la planeación económica y territorial; su carácter holístico, pues, tiene en cuenta tanto los aspectos físicos como sociales de los territorios para su organización y planeación; su multiescalaridad, que busca respuestas no solo a los problemas de los municipios, sino también de toda la zona, el departamento y aun de la región; la larga duración de sus ciclos, que garantiza una planeación a corto, mediano y largo plazo y con lo cual se evita el fraccionamiento de las políticas de planeación y la imposición de modelos diferentes cuando asumen nuevas autoridades municipales. En fin, sin considerarla una panacea, la NPTE puede ayudar a construir espacios más equitativos y sostenibles en el sur del Atlántico.

\section{Referencias}

Alcaldía Municipal de Campo de la Cruz (2016). Plan de desarrollo 2016-2019. Disponible en: http:/campodelacruz-atlantico.gov.co/apc-aafiles/31383838393836663438666137623166/ pdm_2016_2019.pdf

Alcaldía Municipal de Candelaria (2016). Plan de desarrollo 2016-2019. Disponible en: http://candelaria-atlantico.gov.co/apc-aafiles/31313663626230326561323539636338/ plan-de-desarrollo-2016-final.pdf

Alcaldía Municipal de Repelón (S.f) Plan de Ordenamiento Territorial. En: www.repelon-atlantico. gov.coplaneacion. shtml apc $=$ plPlan $\% 20$ de\%20Ordenamiento\%20Territorial$1-\& x=2503054$

Alcaldía Municipal de Santa Lucía (S.f). Esquema de Ordenamiento Territorial. En: http:// santalucia-atlantico.gov.co/planeacion. shtml?apc $=$ plPlan $\% 20 \mathrm{de} \% 20$ Ordenamiento\%20Territorial-1- \&x=1558782

Cámara de Comercio de Barranquilla (2011). Atlántico en cifras 2009. En: http://www.camarabaq.org. co/index.php?option $=$ com_content\&view=arti cle\&id $=225 \&$ Itemid $=186$

Departamento Administrativo Nacional de Estadística. (2011). Censo General 2005. Atlántico. En: http://www.dane.gov.co/files/censo2005/ PERFIL_PDF_CG2005/08000T7T000.PDF

Departamento Administrativo Nacional de Estadística. (2011). Necesidades Básicas Insatisfechas. En: http:/www.dane.gov.co/ daneweb_V09/index.php?option=com_content $\&$ view $=$ article\&id $=231 \&$ Itemid $=66$

Departamento Nacional de Planeación (2011). Agenda Atlántico 2020: La ruta para el desarrollo. Bogotá: Departamento Nacional de Planeación: Gobernación del departamento del Atlántico. Disponible en: https://colaboracion.dnp.gov. co/CDT/Prensa/Publicaciones/7-VISION\%20 ATLANTICO.pdf

Departamento Nacional de Planeación. (2014). Índices de desempeño fiscal por municipios. Bogotá: 
Departamento Nacional de Planeación.

Disponible en: www.dnp.gov.co/programas/ desarrollo-territorial/evaluacion-y-seguimientode-la-descentralizacion/Paginas/desempenofiscal.aspx

El Heraldo. Diario de Barranquilla. Ediciones correspondientes a los meses de noviembre $\mathrm{y}$ diciembre de 2010 y enero de 2011.

Farinós, J. (2009). Bases, métodos e instrumentos para el desarrollo y la cohesión territoriales. En: Farinós, J. Romero, J. y J. Salom, Eds. 2009. Cohesión e Inteligencia territorial. Colección: Desarrollo Territorial. Serie Estudios y Documentos, 7. Publicaciones de la Universidad de Valencia.

Farinós, J. (2010). Nueva planificación territorial. Aproximación conceptual y metodológica. Módulo presentado en el Seminario de Profundización I en el Curso de Doctorado de Geografia del convenio entre la Universidad Pedagógica y Tecnológica de Colombia (UPTC) y el Instituto Geográfico Agustín Codazzi (IGAC). Bogotá, agosto 14, 20 y 21 de 2010.

Farinós, J. y J. Romero. (2007). Territorialidad y buen gobierno para el desarrollo sostenible. Colección: Desarrollo Territorial. Serie Estudios y Documentos, 2. Publicaciones de la Universidad de Valencia.

Fernández, J. (2006). Planificación estratégica de ciudades: Nuevos instrumentos y procesos. Barcelona: Editorial Reverté.

Gobernación del departamento del Atlántico (2012). Análisis de la situación de la salud. Barranquilla: Gobernación del departamento del
Atlántico: Secretaría de salud departamental. Disponible en: https://www.minsalud.gov. co/plandecenal/mapa/Analisis-de-SituacionSalud-Atlantico-2012.pdf

Gómez Orea, D. (2007). Requisitos de la Planificación Territorial hoy. Evaluación de los procesos territoriales. En: Farinós, Jy J. Romero. 2007. Territorialidad y buen gobierno para el desarrollo sostenible. Colección: Desarrollo Territorial. Serie Estudios y Documentos, 2. Publicaciones de la Universidad de Valencia.

Harvey, D. (2003). Espacios de Esperanza. Madrid: Ediciones Akal.

Instituto Colombiano de Fomento de la Educación Superior, ICFES. (2016). Clasificación de planteles. Atlántico. En:http://www.icfesinteractivo.gov.co/Clasificacion/index_ppal_ind.jsf Instituto Colombiano de Fomento de la Educación Superior, ICFES. (2016). Reportes históricos. Disponible en: http://www.icfesinteractivo.gov. co/historicos/

Instituto Geográfico Agustín Codazzi, IGAC. (1994). Atlántico. Características Geográficas. Bogotá: Instituto Geográfico Agustín Codazzi.

Instituto Geográfico Agustín Codazzi (IGAC). (2016). Departamento del Atlántico. División políticoadministrativa. Disponible en: http:/geoportal. igac.gov.co/mapas_de_colombia/igac/ politicos_admin_2014/Atlantico.pdf Iracheta, J. (1997). Planeación y desarrollo: Una visión del futuro. México: Plaza y Valdés Editores.

Llanos, E. (2007). El proceso de transformación espacial de Barranquilla en el siglo XX. En: Perspectiva Geográfica, № 12, año 2007. 
Bogotá: Instituto Geográfico Agustín Codazzi (IGAC) y Universidad Pedagógica y Tecnológica de Colombia (UPTC).

Llanos, E. (2010). Metropolización de Barranquilla y problemática espacial de Soledad (Atlántico). En: Perspectiva Geográfica, № 15, año 2010. Bogotá: Instituto Geográfico Agustín Codazzi (IGAC) y Universidad Pedagógica y Tecnológica de Colombia (UPTC).

Massiris, A. (2009). Desarrollo Territorial Sostenible en América Latina. En: Farinós, J. Romero, J. y J. Salom, Eds. 2009. Cohesión e Inteligencia territorial. Colección: Desarrollo Territorial. Serie Estudios y Documentos, 7. Publicaciones de la Universidad de Valencia.
Navarro, E. (2011). Salud Pública: así estaban los municipios antes del desastre. En: Unirte. Enero de 2011, p. 3. Barranquilla: Publicaciones de la Universidad del Norte.

Peñaloza, M. (2008). Currículo y calidad de la educación. Educación y Humanismo, 10 (15), 189-193.

Sánchez, A. (2011). Después de la inundación. Documentos de Trabajo sobre Economía Regional, No 150. Cartagena: Banco de la República.

Santos, M. (2000). La naturaleza del espacio. Madrid: Ariel.

Tovar, R. (1986). El enfoque geohistórico. Caracas: Academia Nacional de Historia. 